\title{
METODOLOGÍA DE IMPLEMENTACIÓN DEL “PLAN DE EGRESO HOSPITALARIO” PARA PERSONAS CON ENFERMEDAD CRÓNICA EN COLOMBIA
}

\author{
GLORIA MABEL CARRILLO GONZÁLEZ ${ }^{*}$, BEATRIZ SÁNCHEZ HERRERA² \\ OLGA JANETH GÓMEZ ${ }^{3,}$ SONIA PATRICIA CARREÑO ${ }^{4}$, LORENA CHAPARRO DÍAZ ${ }^{5}$ \\ 1* MSc y PhD. Enfermera Profesora Asociada Universidad Nacional de Colombia \\ ${ }^{2}$ MSc. Enfermera Profesora Titular (P) Universidad Nacional de Colombia \\ ${ }^{3} \mathrm{MSc}$ y PhD. Enfermera Profesora Asociada Universidad Nacional de Colombia \\ ${ }^{4} \mathrm{MSc}$ y PhD. Enfermera Profesora Auxiliar Universidad Nacional de Colombia \\ ${ }^{5} \mathrm{PhD}$. Enfermera Profesora Asociada Universidad Nacional de Colombia \\ Correspondencia: *1 gmcarrillog@unal.edu.co
}

Carrera 30 no. 45-01. Edificio 228 oficina 302 bogotá-colombia.

Recibido: 22 Abril 2017 Aceptado: 15 Junio 2017

\section{RESUMEN}

Objetivo: Diseñar y validar una metodología para implementar el "Plan de egreso hospitalario" como mecanismo de apoyo en el cuidado de la salud de personas con enfermedad crónica (EC) y sus cuidadores familiares (CF) en Colombia.

Metodología: Estudio de tipo descriptivo cuyo desarrollo contó con los siguientes pasos:1) Consolidación de una guía. 2) Validación del proceso propuesto para la implementación del "Plan de egreso hospitalario". 3) Prueba piloto de implementación.

Resultados: Con base en el estado del arte y la definición de pasos del cuidado que se requieren para el alta hospitalaria se propone una guía de acción basada en la revisión de las condiciones de la institución hospitalaria y en el nivel de competencia para el cuidado en el hogar de los usuarios, buscando garantizar un cuidado continuo y seguro al salir de la institución.

Conclusiones: La Metodología utilizada para desarrollar el "Plan de egreso hospitalario" para personas con enfermedad crónica en Colombia que se elaboró y validó en el presente estudio aporta elementos conceptuales y de procedimiento para implementar y sistematizar un programa de alta hospitalaria con base en el fortaleciendo de las condiciones institucionales y de la competencia de cuidado por parte de las personas con EC y sus CF. Dicha metodología constituye una herramienta para ayudar a disminuir la carga del cuidado de la EC en Colombia.

Palabras Clave: Alta del paciente; Enfermedad crónica; Cuidados de enfermería en el hogar; Autocuidado; Cuidadores. 


\title{
METHODOLOGY FOR THE IMPLEMENTATION OF THE “HOSPITAL DISCHARGE PLAN” FOR PEO- PLE WITH CHRONIC DISEASE IN COLOMBIA
}

\begin{abstract}
Objective: Design and validate a methodology for the implementation of the "hospital Discharge Plan" as a support mechanism in the health care for patients with chronic disease $(\mathrm{CD})$ and their family caregivers $(\mathrm{CF})$ in Colombia.

Methodology: Descriptive study that is part of the Program for the Reduction of the disease burden in Colombia. For its development, it included the following steps: 1) Consolidation of a guideline. 2) Validation of the "Hospital Discharge Plan" implementation process. 3) Test pilot implementation the "Hospital Discharge Plan"

Results: Based on the state of the art a careful definition of steps required to develop a "Hospital Discharge Plan" are addressed. The core issue is the caring competence of the patient and his /her family caregiver in order to assure continuous and safe care when leaving the institution.

Conclusions: The methodology used to develop the "Hospital Discharge Plan" for people with chronic disease in Colombia that was developed and validated in this study provides conceptual and procedural elements to implement and systematize a discharge program based on the strengthening of the caring competence of the people with $\mathrm{CD}$ and their family caregivers. This methodology is a tool to help reduce the burden of care of people with CD in Colombia.
\end{abstract}

Key Words: Patient Discharge; Chronic Illness; Home Health Nursing; Self-Care; Caregivers.

\section{METODOLOGIA PARA A APLICAÇÃO DO “PLANO DE DESCARGA HOSPITALAR” PARA PESSOAS COM DOENÇA CRÔNICA NA COLÔMBIA}

\section{RESUMO}

Objetivo: Projetar e validar uma metodologia para a implementação do "Plano de descarga hospitalar" como mecanismo de suporte nos cuidados de saúde para pacientes com doença crônica (CD) e seus cuidadores familiares (CF) na Colômbia.

Metodologia: Estudo descritivo que faz parte do Programa de Redução da carga de doença na Colômbia. Para o seu desenvolvimento, incluiu as seguintes etapas: 1) Consolidação de uma diretriz. 2) Validação do processo de implementação do "Plano de descarga hospitalar". 3) Implementação do piloto de teste o "Plano de descarga hospitalar".

Resultados: Com base no estado da arte, uma definição cuidadosa das etapas necessárias para desenvolver um "Plano de descarga hospitalar" são abordadas. A questão central é a competência de atendimento do paciente e seu cuidador familiar, a fim de garantir cuidados contínuos e seguros ao deixar a instituição.

Conclusões: A metodologia utilizada para desenvolver o "Plano de descarga hospitalar" para pessoas com doença crônica na Colômbia que foi desenvolvida e validada neste estudo fornece elementos conceituais e processuais para implementar e sistematizar um programa de alta com base no fortalecimento da competência de cuidar da pessoas com $\mathrm{CD}$ e seus cuidadores familiares. Esta metodologia é uma ferramenta para ajudar a reduzir a carga de atendimento de pessoas com $\mathrm{CD}$ na Colômbia.

Palavras-chave: descarga do paciente; Doença crônica; Home Health Nursing; Cuidados pessoais; Cuidadores. 


\section{Introducción}

La enfermedad crónica (EC) ha sido declarada una prioridad tanto en la atención en salud a nivel mundial (1), como en la investigación en salud en Colombia(2). Este tipo de enfermedad se identifica entre otras cosas, por tener diferentes etapas de remisión y exacerbación de síntomas que duran toda la vida (3). En el transcurso de esas etapas la mayor parte de las personas con EC pueden llegar a necesitar de otro que le acompañe en sus labores y le brinde asistencia básica en todo momento; este es denominado cuidador. En su mayoría el cuidador posee un vínculo familiar o un lazo afectivo de compromiso y es quien se apropia de la responsabilidad del cuidado, participando, asumiendo decisiones y conductas acerca de la persona cuidada(4).

Un cuidador es miembro de una familia o un grupo social muy cercano que asume la responsabilidad y las decisiones de cuidado del ser querido que está enfermo y por lo general representa una solución ante la necesidad de atenderlo. Se ha establecido que es en el cuidador familiar en quien recae el compromiso, moral e ineludible, de velar por el miembro de su familia o amigo cercano enfermo y en consecuencia en quien recae la mayor carga del cuidado (5).

Si bien la carga de la EC ha sido ampliamente documentada, hasta ahora se comienza a hablar de la carga del cuidado que esta genera. Se ha demostrado que se requieren conocimientos y habilidades por parte de los pacientes y de sus cuidadores familiares (CF) (6), para el ejercicio de su rol y que muchas veces no cuentan con ellos.

El apoyo de los profesionales, las instituciones y el sistema de salud debe responder a estas demandas para garantizar el derecho a un cuidado de calidad para la persona enferma y el respaldo requerido por sus $\mathrm{CF}$.

Ahora bien, el cuidado crónico de calidad tiene varias características ampliamente documentadas que incluyen entre otras: el acceso, la seguridad, la continuidad, el soporte social con uso de la tecnología de información y comunicación adecuada y el fortalecimiento de las capacidades de automanejo (7) y competencia para el cuidado (8).

Con base en las anteriores premisas, el presente estudio buscó diseñar y validar una metodología para implementar el "Plan de egreso hospitalario" como mecanismo de apoyo en el cuidado de la salud de personas con enfermedad crónica (EC) y sus cuidadores familiares (CF) en Colombia.

\section{Metodología}

El presente estudio se desarrolló entre los años 2011 2014. Contó con el aval del Comité de ética institucional y mantiene todos requisitos exigidos por la norma 8430 de 1993, por la cual se establecen las pautas científicas, técnicas y administrativas para la investigación en salud en Colombia (9).

se considera descriptivo exploratorio por cuanto diseña y valida una propuesta estructurada de intervención, denominada "Plan de egreso hospitalario", dirigida a personas con EC y a sus CF en Colombia. Con base en una revisión integrativa desarrollada y publicada previamente (10), se cumplieron 3 fases que son descritas a continuación:

- Consolidación de una guía dirigida a enfermería. Con los insumos conceptuales logrados en los pasos anteriores, se avanzó en la consolidación de una Guía, que permitió establecer los pasos requeridos para poder hacer operativo en contexto el "Plan de egreso hospitalario".

- Validación de la guía y el proceso propuesto para la implementación del "Plan de egreso hospitalario". Una vez enunciados los pasos del proceso $e$ identificados los insumos requeridos para que este sea exitoso, su contenido y materiales acompañantes fueron validados por un grupo de expertos que incluyó 7 profesionales del área de la salud con experiencia personal como cuidadores familiares de personas con EC y que además trabajan en el área, 1 psicóloga, 2 estadísticos con experiencia en trabajos de cuidado de enfermería, quienes revisaron el contenido y el formato propuestos para la implementación del plan de egreso hospitalario. En primer lugar, se acudió a validación de contenido (VDC) (11) de los componentes principales de la guía. Los expertos calificaron en la escala tipo Likert con cinco puntos donde 1 es total desacuerdo hasta 5 completamente de acuerdo la claridad, la precisión, la comprensión, la relevancia y la pertinencia del contenido de la guía. Se consideró la nota atribuida para cada uno de los componentes y a partir de esto, el promedio ponderado de las opciones señaladas en la escala Likert con variación de 1 a 5 puntos en donde $1=0$; $2=0,25 ; 3=0,5 ; 4=0,75$ y $5=1$. Promedios mayores o iguales a 0,8 se consideraron "altamente adecuados", entre 0,5 y 0,79 "adecuados" y por debajo de 0,5 "no adecuados" (11). Posteriormente se dejó un espacio para observaciones sobre cada uno de los pasos descritos en la guía.

- Prueba piloto de implementación del programa plan 
de egreso: se adelantó con 120 usuarios de las cinco macro regiones de Colombia para determinar el valor instructivo y predictivo de la metodología propuesta.

- Al clarificar, las condiciones, los roles y los pasos requeridos para hacer operativo el plan de egreso, se procedió a la descripción integral del proceso cuya salida se centró en el fortalecimiento de la competencia de cuidado en el hogar del paciente o su $\mathrm{CF}$, según proceda. Para medir la competencia para cuidar en el hogar se empleó la versión abreviada del instrumento Competencia para el cuidado en el hogar, (GCPC-UN-CPC), en sus versiones pacientes y cuidador familiar. Este instrumento considera que la competencia para cuidar en el hogar es la capacidad, habilidad y preparación que tiene la persona con enfermedad crónica o su cuidador familiar para ejercer la ejercer la labor de cuidar en su sitio de vivienda. La versión original tiene 60 ítems y la abreviada 20, que miden con una escala tipo Likert con puntuaciones de 1 a 4 , donde 4 es el máximo valor posible, seis categorías complementarias entre si y que se encuentran ordenadas bajo el acróstico CUIDAR, así: Conocimiento, Unicidad o condiciones particulares, Instrumental procedimental, Disfrute de condiciones mínimas para el cuidado o nivel de bienestar, Anticipación, y Relación social e interacción. Las dos versiones de este instrumento cuentan con las pruebas psicométricas para ser usadas en Colombia incluidas validez aparente, validez de constructo y confiabilidad $(12,13)$.

\section{Resultados}

1. Consolidación de una guía que consta de cinco recomendaciones orientadas a los profesionales de enfermería, a saber:

- Realizar una valoración integral al paciente y al cuidador familiar desde el ingreso a la institución respecto a: estado mental, funcionalidad, competencia para el cuidado en el hogar (CUIDAR), y ccomplejidad del cuidado asociada al tratamiento en el hogar: polifarmacia, medicamentos parenterales, medicamentos de control y/o dispositivos invasivos.

- Clasificar al usuario según grado de riesgo para el egreso, con el fin de planear la conducta a seguir. (Tabla 1)

- Brindar educación e instrucción al paciente y al cuidador familiar durante la estancia hospitalaria previendo las necesidades de cuidado al momento del egreso.

- Remitir a grupos de apoyo y orientar al paciente y al cuidador familiar (o quien haga sus veces) sobre las interconsultas requeridas.

- Realizar seguimiento hasta por un mes de acuerdo al grado de riesgo determinado. Como mínimos se considera que usuarios de grado de riesgo bajo tengan seguimiento extra institucional de $1 \mathrm{vez}$ por semana; para usuarios de alto riesgo por lo menos 2 a la semana.

Tabla 1. Criterios clasificación del grado de riesgo para el egreso

\begin{tabular}{|c|c|c|c|c|c|}
\hline \multirow{2}{*}{ GRADO DE RIESGO } & \multicolumn{3}{|c|}{$\begin{array}{l}\text { COMPLEJIDAD DEL CUIDADO } \\
\text { ASOCIADO AL TRATAMIENTO }\end{array}$} & $\begin{array}{l}\text { COMPETENCIA PARA EL } \\
\text { CUIDADO EN EL HOGAR } \\
\text { (CUIDAR) }\end{array}$ \\
\cline { 2 - 5 } & $\begin{array}{l}\text { Número de } \\
\text { medicamen- } \\
\text { tos }(>5)\end{array}$ & $\begin{array}{l}\text { Via } \\
\text { de adminis- } \\
\text { tración (Pa- } \\
\text { renteral) }\end{array}$ & $\begin{array}{l}\text { Tipo me- } \\
\text { dicamento } \\
\text { (Control) }\end{array}$ & $\begin{array}{l}\text { Dispositivos } \\
\text { invasivos }\end{array}$ & Media o alta \\
\hline Bajo & No & No & No & No & Baja o media \\
\hline Alto & \multicolumn{2}{|c|}{ Una o más de estas condiciones } & \\
\hline
\end{tabular}

Fuente: Datos del estudio, 2011-2014

2. Validación del proceso propuesto para la implementación del "Plan de egreso hospitalario"

Los puntajes obtenidos en la validación de contenido estuvieron entre 0,67 y 0,94. Se destaca los puntajes obtenidos por encima de 0,8 en las recomendaciones de valoración integral al paciente, clasificar según grado de riesgo, brindar educación e instrucción y realizar seguimiento. La recomendación con puntajes más bajos, dentro del rango de "adecuado" fue la de remisión a grupos de apoyo. (Tabla 2). 
Tabla 2. Puntaje Validez de Contenido Recomendaciones Guía de plan de egreso.

\begin{tabular}{|l|c|c|c|c|c|}
\hline Recomendación /Criterio & Claridad & Precisión & Comprensión & Relevancia & Pertinencia \\
\hline $\begin{array}{l}\text { 1: Realizar valoración integral al pacien- } \\
\text { te y al cuidador }\end{array}$ & 0,80 & 0,83 & 0,83 & 0,88 & 0,80 \\
\hline $\begin{array}{l}\text { 2: Clasificar al usuario según grado de } \\
\text { riesgo para el egreso }\end{array}$ & 0,83 & 0,80 & 0,83 & 0,80 & 0,80 \\
\hline $\begin{array}{l}\text { 3: Brindar educación e instrucción al } \\
\text { paciente y al cuidador }\end{array}$ & 0,86 & 0,86 & 0,91 & 0,88 & 0,88 \\
\hline $\begin{array}{l}\text { 4: Remitir a grupos de apoyo } \\
\text { 5: Realizar seguimiento hasta 1 mes } \\
\text { después del egreso }\end{array}$ & 0,72 & 0,67 & 0,72 & 0,70 & 0,78 \\
\hline
\end{tabular}

Fuente: Datos del estudio.

Se analizaron las sugerencias de los expertos con base en lo cual se establecieron 7 premisas fundamentales a tener en cuenta para la implementación del plan:

a. En la valoración se pretende conocer la condición de la diada Paciente - cuidador familiar. La determinación del sujeto con quien se hace el énfasis, admitiendo que en todo caso la diada está involucrada, depende del nivel de autonomía funcional y cognitiva del paciente de acuerdo con las pruebas de nivel de funcionalidad y el estado mental.

b. En la clasificación del riesgo se determina la complejidad del tratamiento y el nivel de vulnerabilidad del usuario al salir del hospital. La complejidad del tratamiento revisa si tiene 5 o más medicamentos, si hay una vía de administración diferente a la oral, si alguno de los medicamentos indicados se considera un medicamento de control y si sale del hospital con dispositivos invasivos. En cualquiera de estos casos el tratamiento se considera de alto riesgo. Se procede luego a revisar el nivel de competencia para el cuidado en donde el tener una competencia en nivel medio o bajo se considera un caso de alto riesgo.

c. La definición de la conducta depende de los resultados de los pasos anteriores en donde la misma herramienta GCPC-UN-CPC sugiere la conducta a seguir de modo que si hay dificultades de CUIDAR en el Conocimiento, la diada se dirige a educación, si las hay en la Unicidad o condición particular de las personas implicadas estas serán remitidas al servicio específico de apoyo que requieran. Si hay dificultad en el aspecto Instrumental o procedimental, se dirigen a educación y se les debe hacer un seguimiento sobre la instrucción. Si la dificultad se presenta en el campo del Disfrute de condiciones mínimas o nivel básico de bienestar, se deben remitir al servicio o soporte requerido. Si se encentran problemas en la capacidad de Anticipar o prever y manejar las emergencias, se sugiere orientar a educación y hacer un seguimiento de este aspecto y si la dificultad está en el campo de las Relaciones o red de soporte, se debe remitir al servicio requerido para ello. Es necesario tener en cuenta que al momento de definir la conducta los demás profesionales y la institución deben estar preparados para asumirla. En este caso las conductas se pueden concretar en tres: educación, seguimiento y remisión a servicios o grupos de apoyo.

d. De la educación es responsable la enfermera gestora del plan de egreso, la coordinadora del Programa Plan de Egreso y cada una de las enfermeras de los servicios. Requieren como soporte material educativo disponible: folletos, volantes, videos, cartillas y otros materiales que hayan sido validadas por pacientes $y$ por el equipo de salud. 
e. Del seguimiento es responsable la coordinadora del Programa Plan de egreso definiendo si se requiere consulta programada, llamada telefónica o intercambio virtual con apoyo de herramientas informáticas. Se requiere acceso a líneas de teléfono, consultorio de Plan de egreso y computador con internet disponiendo de un correo y página institucional.

f. El reconocimiento de todas las áreas y unidades de apoyo disponible son esenciales en este caso, como también la difusión de la política institucional con el respaldo de la alta dirección.

g. En todos los casos se asume que existe algún nivel de riesgo al salir del hospital por lo cual se implementa una lista de chequeo de mínimos que incluye: revisar si además de que la diada paciente - cuidador familiar esté clasificada de acuerdo con su nivel de riesgo para el egreso esta cuenta con la prescripción médica para el egreso, tiene la orden de controles médicos y seguimiento después de la etapa de hospitalización, tiene el listado de servicios hospitalarios de los que puede disponer, conoce los procedimientos y trámites administrativos para garantizar su cuidado, recibe el material educativo de apoyo con las instrucciones específicas de acuerdo a su condición de salud y si conoce el sistema de seguimiento del que dispone en el programa y que incluye un número telefónico y asesoría de enfermería a demanda.

Se consideró, además, la pertinencia de diseñar una estrategia de capacitación virtual denominada "Cuidado continuo en la atención de personas con enfermedad crónica y sus cuidadores" para orientar la formación de los profesionales.

\section{Prueba piloto de implementación del programa plan de egreso}

El programa se implementó en 120 diadas personas con enfermedad crónica-cuidador familiar, captados y desde el ingreso a la institución de salud. La intervención tuvo una duración de hasta un mes después del egreso. Se realizó capacitación y entrenamiento de un profesional de enfermería para el desarrollo del plan encargado de su implementación en todas las fases descritas.

Se comparó la competencia para cuidar en el hogar pre y pos prueba. Se seleccionó el estadístico T de Student para muestras pareadas, previa verificación de ajuste al modelo Gaussiano mediante el estadístico Kolgomorov Smirnov. A continuación, se presentan los resultados de la prueba:
Tabla 3. Resultados competencia para el cuidado en el hogar pre y post intervención

\begin{tabular}{|l|l|}
\hline $\begin{array}{l}\text { Competencia para el cuidado en el hogar } \\
\text { puntajes posibles }\end{array}$ & 60 a 240 \\
\hline Media pre prueba competencia & 153,93 \\
\hline Media posprueba Competencia & 216,75 \\
\hline Desviación estándar pre prueba & 9,688 \\
\hline Desviación estándar posprueba & 6,202 \\
\hline $\mathrm{t}$ & $-54,373$ \\
\hline gl & 58 \\
\hline Valor p & $<0.001$ \\
\hline
\end{tabular}

Fuente: Datos del estudio.

Se identificó que la competencia para el cuidado en el hogar tanto en el enfermo como en el cuidador, tuvo una diferencia con significancia estadística en la postprueba del estudio, lo que se evidencia en el aumento de la media y menor variabilidad; para el uso clínico es recomendable la versión abreviada, excepto en los casos en que la competencia se encuentre seriamente comprometida en donde la versión original puede ser particularmente útil como guía para la intervención y evaluación.

Respecto a las dimensiones de CUIDAR se evidenciaron cambios estadísticamente significativos en los componentes de Conocimiento, Unicidad Instrumental o procedimental, Disfrute de condiciones mínimas o nivel básico de bienestar y Anticipar o prever y manejar las emergencias. En el de Relaciones o red de soporte, se identificaron cambios mínimos que no fueron estadísticamente significativos.

Se establecieron tres aspectos fundamentales para fortalecer la competencia: el primero, determinar la posibilidad que el paciente tiene de cuidarse o liderar su cuidado definiendo si se enfatiza en su competencia o en caso de no poder hacerlo en la de su cuidador familiar. Segundo, garantizar unos mínimos en el alta hospitalaria para que el paciente y su familia sepan cómo cuidarse. El último, la importancia de clasificar de acuerdo con el nivel de competencia y la complejidad del tratamiento, el tipo de apoyo que requiere el paciente y su CF para garantizar un cuidado seguro y continúo al salir a su hogar. 


\section{Discusión}

Frente a la responsabilidad de cuidado que deben asumir las personas con EC y sus CF con procesos de adaptación permanente, participación y negociación en medio de la rutina, es necesario garantizar la continuidad, seguridad e integralidad de dicho cuidado.

La EC se caracteriza por ir en incremento y mantenerse en el tiempo y en tal sentido, las relaciones de cuidado deben ser analizadas en diadas CF - receptor del cuidado, las cuales surgen como una opción del desarrollo humano, en las que se requieren de dos personas o en el caso del auto-cuidado, un diálogo interno con el propio ser, tal como ha sido descrito en población colombiana(14). Una vez inicia la dependencia funcional se van generando acciones mutuas a partir de un contrato que en la mayor parte de los casos es tácito y que llevan a tener mutualidad y reciprocidad que generan y mantienen un vínculo especial(15).

El presente estudio ratifica la importancia y pertinencia de desarrollar planes de transición y egreso como elemento fundamental en el cuidado de los pacientes y de sus cuidadores familiares(16-21). Se evidencia la necesidad de apoyar de manera operativa la implementación de una política nacional. El aporte del instrumento GCPC- UN$\mathrm{CPC}$ como lineamiento de los mínimos, puede servir de orientación para su estructuración ya que este complementa los desarrollos actuales (22-25) y a diferencia de estos, cumple con las características de integralidad, seguridad y continuidad, además de tener sus pruebas psicométricas en español.

El eje de la atención en el usuario mediante la competencia de cuidar en el hogar aporta a los estudios de conceptualización de los elementos que debe tener un plan de egreso hospitalario y apoya el cuidado en un momento de alta vulnerabilidad para el paciente y su cuidador familiar como es el egreso hospitalario (26-29). Las pautas para fortalecer la competencia de cuidar en el hogar pueden ser un aporte singular para cualificar la práctica. Esta metodología tiene potencial para apoyar y sistematizar la implementación del plan de egreso a nivel institucional aumentando así los beneficios y beneficiarios de la atención en salud (30-31).

El empleo las tecnologías de la información y la comunicación (TIC's) para el apoyo y capacitación de los profesionales, se considera de mayor utilidad (32) y genera un panorama prometedor para el mejoramiento permanente del cuidado al paciente crónico y su familia(33), en especial en la región de América Latina(34).

\section{Conclusiones}

La Metodología de implementación del "Plan de egreso hospitalario" para personas con enfermedad crónica en Colombia que se elaboró y validó en el presente estudio aporta elementos conceptuales y de procedimiento para implementar y sistematizar un programa de alta hospitalaria con base en el fortaleciendo de las condiciones institucionales y de la competencia de cuidado por parte de las personas con EC y sus CF. Dicha metodología constituye una herramienta para ayudar a disminuir la carga del cuidado de la EC en Colombia que puede ser replicada en diferentes países de la región de América Latina.

Financiación: Programa para disminuir la carga de la enfermedad crónica en Colombia.

Convocatoria 537- 2010 Colciencias. Unión Temporal Carga de la Enfermedad Crónica en Colombia

Conflicto de intereses: Ninguno

\section{Referencias}

1. OMS.Informesobrelasituaciónmundialdelasenfermedades no trasmisibles 2014 [Dec 13 2014]. Available from: http://apps.who.int/iris/bitstream/10665/149296/1/ WHO_NMH_NVI_15.1_spa.pdf?ua $=1$.

2. Agudelo CA, de la Hoz F, Mojica MJ, Eslava JC, Robledo $\mathrm{R}$, Cifuentes $\mathrm{P}$, et al. Prioridades de investigación en salud en Colombia: perspectiva de los investigadores. Revista de salud pública. 2009;11(2):301-9.

3. Hyman RB, Corbin JM. Chronic illness: Research and theory for nursing practice: Springer Publishing Company; 2001.

4. Barrera L, Pinto N, Sánchez B. Hacia la construcción de un modelo de cuidado de cuidadores de personas con enfermedad crónica. Actualizaciones en Enfermería. 2008;11(2):22-8.

5. Guzmán FJB, Fermán IAT, Nava FV, Gómez CB, Ochoa $\mathrm{DL}$, Torres AAB, et al. Calidad de vida en cuidadores de enfermos asmáticos: un estudio comparativo. Psicología y Salud. 2013;18(2): 181-7.

6. Sánchez-Herrera B, Carrillo-González GM, Barrera-Ortiz L, Chaparro-Díaz L. Carga del cuidado de la enfermedad crónica no transmisible. Aquichan. 2013;13(2):247-60.

7. Organización Mundial de la Salud. Cuidados innovadores para las condiciones crónicas. 2013. Disponible en: http://www2. paho.org/hq/index.php?option=com_docman\&task=doc view\&gid $=22257+\&$ Itemid $=270$ \&lang $=e s$

8. Barrera L, Carrillo G, Chaparro L, Sánchez B. Modelo para abordar la carga del cuidado de la enfermedad crónica en Colombia. Orinoquía. 2015; 19(1): 89-99

9. Ministerio de Salud. Resolución 008430 de 1993 Por la cual se establecen las normas científicas, técnicas y admi- 
nistrativas para la investigación en salud. Ministerio de salud [internet]. 1993[citado 2 Feb 2014]. Disponible en: http:// www.unisabana.edu.co/fileadmin/Documentos/Investigacion/comite_de_etica/Res_8430_1993_-_Salud.pdf

10. Sánchez B, Carrillo GM, Barrera L. El plan de transición y egreso hospitalario y su efecto en el cuidado de la salud: una revisión integrada. Rev. U.D.C.A. Act. Div.Cient. 2014; 17(1): 13-23

11. Fehring R. Methods to validate nursing diagnosis. Heart Lung. 1987;16(6):625-629.

12. Carrillo G, Sánchez B. Vargas E. Desarrollo y pruebas psicométricas del instrumento CUIDAR- versión corta para medir la competencia de cuidado en el hogar. Rev Unv Ind Santander Salud. 2016: 48(2):222-231

13. Carrillo G, Sánchez B, Arias M. Validación de un instrumento para la evaluación de la competencia de cuidado en el hogar del cuidador familiar de una persona con enfermedad crónica. Invest Educ Enferm. 2015; 33(3): 449-455

14. Thompson L, Walker AJ. The dyad as the unit of analysis: Conceptual and methodological issues. Journal of Marriage and the Family. 1982:889-900.

15. Chaparro L. Cómo se constituye el "vínculo especial" de cuidado entre la persona con enfermedad crónica y el cuidador familiar. Aquichan. 2011;11(1):7-22.

16. Lin F, Chaboyer W, Wallis M. A literature review of organisational, individual and teamwork factors contributing to the ICU discharge process. Australian Critical Care. 2009;22(1):29-43.

17. Chen J, Ross JS, Carlson MD, Lin Z, Normand S-LT, Bernheim SM, et al. Skilled nursing facility referral and hospital readmission rates after heart failure or myocardial infarction. The American journal of medicine. 2012;125(1):100. e1-. e9.

18. Han C-Y, Barnard A, Chapman H. Discharge planning in the emergency department: a comprehensive approach. Journal of Emergency Nursing. 2009;35(6):525-7.

19. Rhudy LM, Holland DE, Bowles KH. Illuminating hospital discharge planning: staff nurse decision making. Applied Nursing Research. 2010;23(4):198-206.

20. Hanratty B, Holmes L, Lowson E, Grande G, AddingtonHall J, Payne S, et al. Older adults' experiences of transitions between care settings at the end of life in England: a qualitative interview study. Journal of pain and symptom management. 2012;44(1):74-83.

21. Han C-Y, Chang C-Y, Lee H-L. Another compulsory clinical competence for emergency nurses-Implementing effective discharge planning. Australasian Emergency Nursing Journal. 2009;12(4):178.

22. Kenner C, Boykova M. Transition to home: family perspectives on care in Russia. Newborn and Infant
Nursing Reviews. 2007;7(1):20-4.

23. Senarat U, Gunawardena NS. Development of an instrument to measure patient perception of the quality of nursing care and related hospital services at the National Hospital of Sri Lanka. Asian nursing research. 2011;5(2):71-80.

24. Bobay KL, Jerofke TA, Weiss ME, Yakusheva O. Agerelated differences in perception of quality of discharge teaching and readiness for hospital discharge. Geriatric Nursing. 2010;31(3):178-87.

25. Hadjistavropoulos HD, Garratt S, Janzen JA, BourgaultFagnou MD, Spice K. Development and evaluation of a Continuity of Care Checklist for improving orthopaedic patient discharge from hospital. Journal of Orthopaedic Nursing. 2009; 13(4):183-93.

26. Lowson E, Hanratty B, Holmes L, Addington-Hall J, Grande G, Payne S, et al. From 'conductor'to 'second fiddle': Older adult care recipients' perspectives on transitions in family caring at hospital admission. International journal of nursing studies. 2013;50(9):1197-205.

27. Betz CL. Health care transition for adolescents with special healthcare needs: Where is nursing? Nursing outlook. 2013;61(5):258-65.

28. McBride M, Andrews GJ. The transition from acute care to home: a review of issues in discharge teaching and a framework for better practice. Canadian journal of cardiovascular nursing $=$ Journal canadien en soins infirmiers cardio-vasculaires. 2012;23(3):18-24.

29. Thraen I, Bair B, Mullin S, Weir CR. Characterizing "information transfer" by using a Joint Cognitive Systems model to improve continuity of care in the aged. International journal of medical informatics. 2012;81(7):435-41.

30. Ben-Morderchai B, Herman A, Kerzman H, Irony A. Structured discharge education improves early outcome in orthopedic patients. International Journal of Orthopaedic and Trauma Nursing. 2010;14(2):66-74.

31. Koelling TM, Johnson ML, Cody RJ, Aaronson KD. Discharge education improves clinical outcomes in patients with chronic heart failure. Circulation. 2005;111(2):17985.

32. Coltell O. Servicio Web como soporte de Investigación de las redes de Investigación en Biomédica. La Información de la Salud: Punto de encuentro Disciplinas Sanitarias. 2003.

33. Struk C, Moss J. Focus on technology: What can you do to move the vision forward? Computers Informatics Nursing. 2009;27(3):192-4.

34. Hilbert MR, Bustos S, Ferraz JC. Estrategias nacionales para la sociedad de la información en América Latina y el Caribe. 2005. 